\title{
Equation-of-Motion Coupled-Cluster Theory to Model L-Edge X-ray Absorption and Photoelectron Spectra
}

\author{
Vidal, Marta Lopez; Pokhilko, Pavel; Krylov, Anna I.; Coriani, Sonia
}

Published in:

Journal of Physical Chemistry Letters

Link to article, DOI:

10.1021/acs.jpclett.0c02027

Publication date:

2020

Document Version

Peer reviewed version

Link back to DTU Orbit

Citation (APA):

Vidal, M. L., Pokhilko, P., Krylov, A. I., \& Coriani, S. (2020). Equation-of-Motion Coupled-Cluster Theory to Model L-Edge X-ray Absorption and Photoelectron Spectra. Journal of Physical Chemistry Letters, 11, 83148321. https://doi.org/10.1021/acs.jpclett.0c02027

\section{General rights}

Copyright and moral rights for the publications made accessible in the public portal are retained by the authors and/or other copyright owners and it is a condition of accessing publications that users recognise and abide by the legal requirements associated with these rights.

- Users may download and print one copy of any publication from the public portal for the purpose of private study or research.

- You may not further distribute the material or use it for any profit-making activity or commercial gain

- You may freely distribute the URL identifying the publication in the public portal 


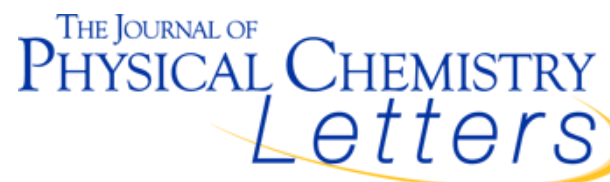

\section{Physical Insights into Light Interacting with Matter}

\section{Equation-of-Motion Coupled-Cluster Theory to Model L-edge X-Ray Absorption and Photoelectron Spectra

\author{
Marta López Vidal, Pavel Pokhilko, Anna I. Krylov, and Sonia Coriani
}

J. Phys. Chem. Lett., Just Accepted Manuscript • DOI: 10.1021/acs.jpclett.0c02027 • Publication Date (Web): 08 Sep 2020

Downloaded from pubs.acs.org on September 15, 2020

\section{Just Accepted}

"Just Accepted" manuscripts have been peer-reviewed and accepted for publication. They are posted online prior to technical editing, formatting for publication and author proofing. The American Chemical Society provides "Just Accepted" as a service to the research community to expedite the dissemination of scientific material as soon as possible after acceptance. "Just Accepted" manuscripts appear in full in PDF format accompanied by an HTML abstract. "Just Accepted" manuscripts have been fully peer reviewed, but should not be considered the official version of record. They are citable by the Digital Object Identifier (DOI®). "Just Accepted" is an optional service offered to authors. Therefore, the "Just Accepted" Web site may not include all articles that will be published in the journal. After a manuscript is technically edited and formatted, it will be removed from the "Just Accepted" Web site and published as an ASAP article. Note that technical editing may introduce minor changes to the manuscript text and/or graphics which could affect content, and all legal disclaimers and ethical guidelines that apply to the journal pertain. ACS cannot be held responsible for errors or consequences arising from the use of information contained in these "Just Accepted" manuscripts. 


\title{
Equation-of-Motion Coupled-Cluster Theory to Model L-edge X-Ray Absorption and Photoelectron Spectra
}

\author{
Marta L. Vidal, ${ }^{*, \dagger}$ Pavel Pokhilko,,$+\uparrow$ Anna I. Krylov, ${ }^{\ddagger}$ and Sonia Coriani ${ }^{*, \dagger}$ \\ $\dagger D T U$ Chemistry - Department of Chemistry, Technical University of Denmark, \\ Kemitorvet Bldg 207, DK-2800, Kongens Lyngby, Denmark \\ $\ddagger$ Department of Chemistry, University of Southern California, Los Angeles, California \\ 90089-0482 \\ I Current address: Department of Chemistry, University of Michigan, Ann Arbor, MI \\ 48109, USA
}

E-mail: malop@kemi.dtu.dk; soco@kemi.dtu.dk 


\begin{abstract}
We present an extension of the equation-of-motion coupled-cluster singles and doubles (EOM-CCSD) theory for computing x-ray L-edge spectra, both in the absorption (XAS) and photoelectron (XPS) regimes. The approach is based on the perturbative evaluation of spin-orbit couplings using the Breit-Pauli Hamiltonian and nonrelativistic wave-functions described by the fc-CVS-EOM-CCSD ansatz (EOM-CCSD within the frozen-core core-valence separated (fc-CVS) scheme). The formalism is based on spinless one-particle density matrices. The approach is illustrated by modeling XAS and XPS of several model systems ranging from argon atoms to small molecules containing sulfur and silicon.
\end{abstract}

\title{
Graphical TOC Entry
}

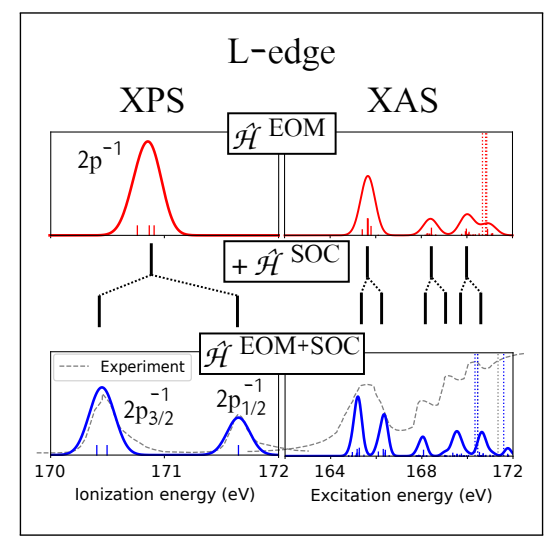

\section{Keywords}

Inner-shell 2p spectra, spin-orbit coupling, coupled-cluster theory, equation-of-motion, corevalence separation, Breit-Pauli Hamiltonian, Dyson orbitals 
Spectroscopic techniques exploiting x-ray radiation have a long history. Two of the most popular ones, x-ray absorption (XAS) and x-ray photoemission (XPS, also known as x-ray photoelectron or electron spectroscopy for chemical analysis, ESCA), enable investigation of the local electronic structure in molecules and materials. Todays light sources, which range from synchrotron and x-ray free-electron lasers to table-top x-ray instruments based on high harmonic generation, facilitate exciting new experiments, which were merely hypothetical just a few years back. These advances have triggered an explosion of interest of the molecular and material sciences community in x-ray based techniques. ${ }^{1-3}$ These advances in the experimental tools have been accompanied by a burst of activity in the development of theoretical methods for simulating and interpreting experimental spectra. ${ }^{4}$

Conceptually, x-ray spectroscopy is similar to UV-Vis spectroscopy, the main difference being the energy scale and, consequently, the type of electronic transitions that are probed. $\mathrm{UV}-\mathrm{V}$ is radiation induces transitions involving the outer-shell valence electrons, whereas $\mathrm{x}$ ray radiation induces transitions involving inner-shell core electrons. Despite this similarity, the theoretical methods developed for valence spectroscopy are not directly applicable to core-level spectroscopies. ${ }^{4}$ Similarly to their valence counterparts, core-level states often have open-shell character, but they also exhibit strong orbital relaxation. Thus, their description requires sufficiently flexible basis sets ${ }^{5,6}$ and many-body ansätze that are capable of tackling static and dynamic correlation as well as orbital relaxation.

The equation-of-motion (EOM) coupled-cluster (CC) framework ${ }^{7-12}$ is a versatile platform for treating excited and ionized states with open-shell character. Even at the lowest level of correlation treatment, when only single and double excitations are included in the ansatz, the method has the ability to tackle both dynamic correlation and orbital relaxation quite well. Originally, EOM-CC was developed to study valence states; thus, typical implementations seek the solutions corresponding to the lowest states, which is obviously not suitable for high-energy core-level states. Another complication arises from the fact that the core-level states are embedded in a continuum of valence excited and ionized states, 
which leads to poor convergence and erratic results. ${ }^{13,14}$ The core-valence separation (CVS) scheme, ${ }^{15}$ which decouples the valence and core sectors of the Fock space on the basis of the large energy gap between the core and valence orbitals, provides a simple yet effective recipe for extending valence-state methods into the core-level domain and overcoming the convergence issues. CVS has been implemented within various electronic structure methods, ${ }^{16-21}$ including the EOM-CC family. ${ }^{22-24}$ The resulting methods have been successfully applied to model a variety of $\mathrm{x}$-ray spectroscopic experiments such as absorption (XAS), ${ }^{22,23,25,26}$ photoelectron (XPS), ${ }^{24,27,28}$ X-ray emission (XES), and resonant inelastic scattering (RIXS). ${ }^{14,29-31}$ At the CC level, the applications have been so far limited to the study of $1 \mathrm{~s}$ electrons of light elements (that is, the K-edge), due to yet another obstacle towards the quantitative simulation of x-ray spectra - the need to include relativistic effects.

Relativistic effects, which become more pronounced at higher energies, can be classified into two categories: scalar and spin-orbit. ${ }^{32}$ The first type is not critically important in the context of K-edge spectroscopy, because it results in a constant energy shift of the entire spectrum. ${ }^{5,27,33}$ Because K-edge states are mostly affected by scalar relativistic effects, non-relativistic calculations yield qualitatively correct K-edge spectra. The second type, spin-orbit coupling (SOC), which arises from the coupling between the magnetic moment associated with the spin of the electron and the magnetic field created by the relative motion of charged particles (electrons and nuclei), ${ }^{34}$ has a greater impact on the spectra at lower edges. SOC mixes states with different multiplicity, which do not interact in a non-relativistic framework. It also causes energy splittings of orbitals with nonzero orbital angular momentum $(l>0)$. Fig. 1 shows the SO splitting of the atomic $2 p$ orbitals, illustrating that SOC affects the L-edge spectra by splitting them into two edges: $\mathrm{L}_{2}\left(\right.$ or $\left.\mathrm{L}_{I I}\right)$ and $\mathrm{L}_{3}$ (or $\mathrm{L}_{I I I}$ ). 


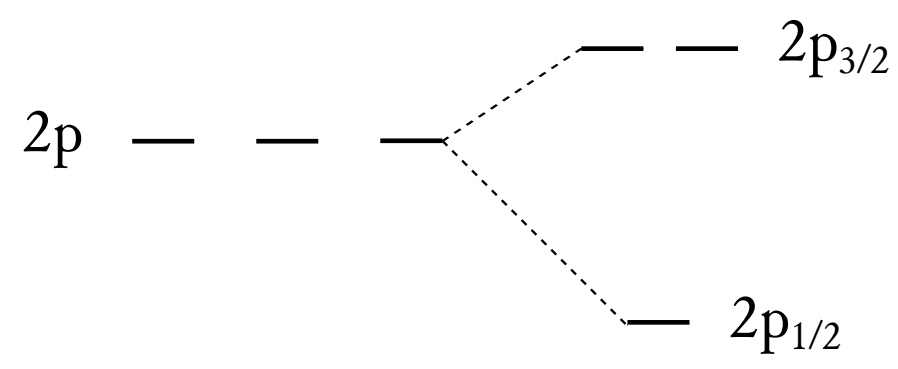

Figure 1: Splitting of the $2 p$ orbitals due to spin-orbit coupling.

These effects can be fully accounted for within a fully relativistic treatment with a 4component Hamiltonian, ${ }^{35-37}$ but such treatments come with a substantial increase of the computational cost. Various flavors of two-component methods, such as the zeroth-order regular approximation (ZORA) ${ }^{38-41}$ and its infinite-order variant (IORA), ${ }^{42}$ the DouglasKroll-Hess method, ${ }^{43-45}$ or the exact 2-component (X2C) approach, ${ }^{46,47}$ are less demanding; however, these calculations still increase the computational cost by an order of magnitude relative to the non-relativistic calculation. ${ }^{48}$ Furthermore, a variational treatment of the SOC (i.e., when the spin-orbit operator is included at the wave-function optimization step) may lead to an imbalanced description of electronic states with different spin projections, resulting in the violation of Kramers' theorem and momentum contamination. ${ }^{49}$

Fortunately, in molecules composed of atoms from the first few rows of the periodic table, so-called perturbative treatment of SOC, which entails calculation of the matrix elements of the Breit-Pauli Hamiltonian using non-relativistic wave-functions, is sufficiently accurate while being computationally affordable. ${ }^{50}$ This strategy, which has been successfully used within EOM-CC framework, ${ }^{48,51-55}$ has not yet been extended to core-level spectroscopy, which is the focus of this communication. Building upon our previous work, ${ }^{23,24,54-56}$ we implemented calculations of SOC within the frozen core (fc) CVS-EOM-CCSD method, thus extending the EOM-CC framework to modeling x-ray absorption and photoelectron spectroscopy at the L and higher (i.e., lower in energy domain) edges.

In this approach, the final states and their properties (energies and transition strengths) 
are obtained in a two-step procedure. In the first step, the non-relativistic states are computed using the fc-CVS-EOM-CCSD ansatz:

$$
\left|\Psi_{\mathrm{fc}-\mathrm{CVS}-\mathrm{EOM}}\right\rangle=\hat{\mathcal{R}}_{\mathrm{CVS}} e^{\hat{\mathcal{T}}_{\mathrm{fc}}}\left|\Phi_{0}\right\rangle ; \quad \hat{\mathcal{T}}_{\mathrm{fc}}=\sum_{\mu_{v}} t_{\mu_{v}} \hat{\tau}_{\mu_{v}} ; \quad \hat{\mathcal{R}}_{\mathrm{CVS}}=\sum_{\mu_{c}} r_{\mu_{c}} \hat{\tau}_{\mu_{c}}
$$

where $\hat{\mathcal{T}}$ and $\hat{\mathcal{R}}$ are the cluster and the EOM excitation operators, and the sub-indices $v$ and $c$ refer to the valence and core orbital spaces, respectively. The exact form of the $\hat{\mathcal{R}}$ operator depends on the target-state manifold, giving rise to different flavors of EOM-CC methods. ${ }^{9}$ Here, we use EOM-IP (EOM for ionization potentials) to compute ionized states, EOMEE (EOM for excitation energies) to compute singlet excited states, and EOM-SF (EOM spin-flip) to compute triplet excited states (with spin projection $m_{s}=-1$ ). For closed-shell systems, such as those studied here, these calculations employ closed-shell reference states.

In the second step, SOCs are calculated as the matrix elements of the spin-orbit part of the Breit-Pauli Hamiltonian, given in atomic units by: ${ }^{57}$

$$
\hat{\mathcal{H}}^{\mathrm{SO}}=\frac{1}{2 c^{2}}\left(\sum_{i} \mathbf{h}^{\mathrm{SO}}(i) \cdot \mathbf{s}(i)-\sum_{i \neq j} \mathbf{h}^{\mathrm{SO}}(i, j) \cdot(\mathbf{s}(i)+2 \mathbf{s}(j))\right),
$$

where $\mathbf{h}^{\mathrm{SO}}(i)$ and $\mathbf{h}^{\mathrm{SO}}(i, j)$ are the one- and two-electron operators ${ }^{58}$

$$
\begin{gathered}
\mathbf{h}^{\mathrm{SO}}(i)=\sum_{K} \frac{Z_{K}\left(\mathbf{r}_{i}-\mathbf{R}_{K}\right) \times \mathbf{p}_{i}}{\left|\mathbf{r}_{i}-\mathbf{R}_{K}\right|^{3}}=\sum_{K} \frac{Z_{K}}{r_{i K}^{3}}\left(\mathbf{r}_{i K} \times \mathbf{p}_{i}\right)=\sum_{K} \frac{Z_{K}}{r_{i K}^{3}} \mathbf{l}_{i K}, \\
\mathbf{h}^{\mathrm{SO}}(i, j)=\frac{\left(\mathbf{r}_{i}-\mathbf{r}_{j}\right) \times \mathbf{p}_{i}}{\left|\mathbf{r}_{i}-\mathbf{r}_{j}\right|^{3}}=\frac{1}{r_{i j}^{3}} \mathbf{r}_{i j} \times \mathbf{p}_{i}=\frac{1}{r_{i j}^{3}} \mathbf{l}_{i j}
\end{gathered}
$$

$\mathbf{r}_{i}, \mathbf{p}_{i}$ and $\mathbf{l}_{i}$ are the coordinates and the (linear and angular) momenta, respectively, of electron $i$, and $\mathbf{R}_{K}$ and $Z_{K}$ are the coordinates and nuclear charge of nucleus $K$. In second quantization, Eq. (2) assumes the following form: ${ }^{54}$

$$
\hat{\mathcal{H}}^{\mathrm{SO}}=\frac{1}{2 c^{2}}\left(\sum_{p q} I_{p q} \hat{a}_{p}^{\dagger} \hat{a}_{q}+\frac{1}{2} \sum_{p q r s} J_{p q r s} \hat{a}_{p}^{\dagger} \hat{a}_{q}^{\dagger} \hat{a}_{s} \hat{a}_{r}\right)
$$


where $I$ and $J$ refer to the one- and two-electron spin-orbit integrals:

$$
\begin{gathered}
I_{p q}=\left\langle\phi_{p}(1)\left|\mathbf{h}^{\mathrm{SO}}(1) \cdot \mathbf{s}(1)\right| \phi_{q}(1)\right\rangle \\
J_{p q r s}=-\left\langle\phi_{p}(1) \phi_{q}(2)\left|\mathbf{h}^{\mathrm{SO}}(1,2) \cdot[\mathbf{s}(1)+2 \mathbf{s}(2)]\right| \phi_{r}(1) \phi_{s}(2)\right\rangle .
\end{gathered}
$$

Matrix elements of this operator can be computed by contracting the spin-orbit integrals with the corresponding one- and two-electron transition density matrices (TDM):

$$
\left\langle\Psi\left(s, m_{s}\right)\left|\hat{\mathcal{H}}^{\mathrm{SO}}\right| \Psi^{\prime}\left(s^{\prime}, m_{s}^{\prime}\right)\right\rangle=\sum_{p q} I_{p q} \gamma_{p q}+\frac{1}{2} \sum_{p q r s} J_{p q r s} \Gamma_{p q r s}
$$

where

$$
\begin{gathered}
\gamma_{p q}=\left\langle\Psi\left(s, m_{s}\right)\left|\hat{a}_{p}^{\dagger} \hat{a}_{q}\right| \Psi^{\prime}\left(s^{\prime}, m_{s}^{\prime}\right)\right\rangle, \\
\Gamma_{p q r s}=\left\langle\Psi\left(s, m_{s}\right)\left|\hat{a}_{p}^{\dagger} \hat{a}_{q}^{\dagger} \hat{a}_{s} \hat{a}_{r}\right| \Psi^{\prime}\left(s^{\prime}, m_{s}^{\prime}\right)\right\rangle .
\end{gathered}
$$

The calculation of the SOCs, as defined by Eq. (2), entails computationally demanding two-electron part of $\hat{\mathcal{H}}^{\mathrm{SO}}$. Fortunately, the two-electron contributions can be effectively evaluated in a mean-field manner. Within this approximation, called the spin-orbit meanfield $(\mathrm{SOMF}),{ }^{59}$ the calculation of SOC requires only the one-electron TDM

$$
\left\langle\Psi\left(s, m_{s}\right)\left|\hat{\mathcal{H}}^{\mathrm{SOMF}}\right| \Psi^{\prime}\left(s^{\prime}, m_{s}^{\prime}\right)\right\rangle=\sum_{p q} H_{p q}^{\mathrm{SOMF}} \gamma_{p q}
$$

with the following effective one-electron operator $\hat{\mathcal{H}}^{\mathrm{SOMF}}: 51,52,54$

$$
H_{p q}^{\mathrm{SOMF}}=I_{p q}+\frac{1}{2} \sum_{r s} \rho_{r s}\left(J_{p r q s}-J_{p r s q}-J_{r p q s}\right)
$$

where $\rho$ is the state density matrix of the reference determinant. The SOMF approximation simply entails neglecting the non-separable part of two-electron transition density matrix. ${ }^{54}$ By converting these equations into the atomic orbital basis, one can evaluate the SOMF 
integrals using efficient algorithms, such as those used for the Fock-matrix builds. The SOMF Hamiltonian can be used as a starting point for more drastic approximations, such as atomic mean-field approximation in which only the diagonal blocks of $\rho$ (in the AO basis) are retained, and introducing one-center approximations for the one and two-electron integrals. ${ }^{60}$ In our implementation, we used the SOMF scheme without further approximations.

To obtain the SO-split states, we then construct the SOMF Hamiltonian matrix in the basis of zeroth-order states

$$
\mathbf{H}^{\mathrm{SOMF}}=\left\langle\Psi\left(s, m_{s}\right)\left|\hat{\mathcal{H}}^{\mathrm{SOMF}}\right| \Psi^{\prime}\left(s^{\prime}, m_{s}^{\prime}\right)\right\rangle
$$

and diagonalize it. (Here and below we use bold font to denote the matrix representations of the operators expressed in the basis of EOM states). The diagonal elements of the SOMF matrix contain the zeroth-order energies (i.e., non-relativistic EOM-CC energies) and the off-diagonal elements between two different states $\Psi\left(s, m_{s}\right)$ and $\Psi^{\prime}\left(s^{\prime}, m_{s}^{\prime}\right)$ are $\left\langle\Psi\left(s, m_{s}\right)\left|\hat{\mathcal{H}}^{\mathrm{SOMF}}\right| \Psi^{\prime}\left(s^{\prime}, m_{s}^{\prime}\right)\right\rangle$, which are computed as described in Ref. 55. The states entering these expressions are the ionized states $\left(s=1 / 2, m_{s}= \pm 1 / 2\right)$ when we calculate ionization energies, and the singlet $\left(s=0, m_{s}=0\right)$ and triplet $\left(s=1, m_{s}=-1,0,+1\right)$ excited states when we calculate excitation energies. The diagonalization of the SOMF Hamiltonian matrix yields the target SO-coupled states, whose eigenvalues are the energies of the SO-split states. To compute oscillator strengths for the transitions involving the SO-split states, as needed for the simulation of L-edge NEXAFS, we first construct the (non-Hermitian) electric dipole matrices between the ground state $\Psi_{0}$ and the zeroth-order target states $\Psi\left(s, m_{s}\right)$ :

$$
\boldsymbol{\mu}_{\alpha}^{f \leftarrow 0}=\left\langle\Psi^{f}\left(s, m_{s}\right)\left|\hat{\mu}_{\alpha}\right| \Psi_{0}\right\rangle ; \quad \boldsymbol{\mu}_{\alpha}^{0 \leftarrow f}=\left\langle\Psi_{0}\left|\hat{\mu}_{\alpha}\right| \Psi^{f}\left(s, m_{s}\right)\right\rangle,
$$

where $\alpha$ denotes the Cartesian components $x, y$, and $z$. These transition matrices are then transformed into the new basis of the SO-split states by applying the transformation obtained 
from the diagonalization of $\mathbf{H}^{\mathrm{SOMF}}$ :

$$
\tilde{\boldsymbol{\mu}}_{\alpha}^{2}=\mathbf{U}^{\dagger} \boldsymbol{\mu}_{\alpha}^{f \leftarrow 0} \boldsymbol{\mu}_{\alpha}^{0 \leftarrow f} \mathbf{U}
$$

where the matrix $\mathbf{U}$ contains the eigenvectors of $\mathbf{H}^{\mathrm{SOMF}}$. (Due to non-Hermiticity of the EOM theory, the geometric averaging of the estimates from Eq. (15) does not necessarily yield a real positive number; however, for the systems considered here, only very few nonpositive values were observed and their magnitude was negligible.) Finally, the oscillator strengths for the transitions between the ground state and the target SO-coupled state $\tilde{f}$ are computed as:

$$
f(\tilde{f} \leftarrow 0)=\frac{2}{3} \omega_{\tilde{f}} \sum_{\alpha=x, y, z} \tilde{\boldsymbol{\mu}}_{\alpha}^{2} .
$$

In a similar fashion, the XPS intensities can be estimated from the squared norm of the Dyson orbitals, defined as overlap of the initial $N$-electron wavefunction of the neutral and the final $(N-1)$-electron wavefunction of the cation. For the XPS of ground-state species, the initial wavefunction is $\Psi_{0}$ and the final (SO-split) wavefunction is $\Psi_{\tilde{f}}$. Since in CC theory the bra and ket states are not Hermitian conjugates of each other, we use an additional index $L$ or $R$ to mark left and right wavefunctions and the respective Dyson orbitals ${ }^{24,61-63}$

$$
\phi_{\tilde{f}}^{d, R}=\sum_{p} \tilde{\gamma}_{p}^{R, \tilde{f}} \phi_{p} ; \quad \phi_{\tilde{f}}^{d, L}=\sum_{p} \tilde{\gamma}_{p}^{L, \tilde{f}} \phi_{p}
$$

where

$$
\tilde{\gamma}_{p}^{R, \tilde{f}}=\left\langle\Psi_{0}^{L}\left|\hat{a}_{p}^{\dagger}\right| \Psi_{\tilde{f}}^{R}\right\rangle ; \quad \tilde{\gamma}_{p}^{L, \tilde{f}}=\left\langle\Psi_{\tilde{f}}^{L}\left|\hat{a}_{p}\right| \Psi_{0}^{R}\right\rangle
$$

are the expansion coefficients (or amplitudes) of the left $(L)$ and right $(R)$ Dyson orbitals corresponding to the SO-mixed states on the molecular orbital basis $\left\{\phi_{p}\right\}$. The target SO- 
split state $\Psi_{\tilde{f}}$ is the linear combination of the non-relativistic cationic states $\Psi_{j}$

$$
\Psi_{\tilde{f}}^{R}=\sum_{j} \Psi_{j}^{R} U_{j \tilde{f}} ; \quad \Psi_{\tilde{f}}^{L}=\sum_{j} U_{\tilde{f} j}^{*} \Psi_{j}^{L}
$$

so that the left and right Dyson orbitals for the SO-split target state $\Psi_{\tilde{f}}$ become

$$
\begin{aligned}
& \phi_{\tilde{f}}^{d, R}=\sum_{j, p}\left\langle\Psi_{0}^{L}\left|\hat{a}_{p}^{\dagger}\right| \Psi_{j}^{R}\right\rangle U_{j \tilde{f}} \phi_{p}=\sum_{p}\left(\sum_{j} \gamma_{p}^{R, j} U_{j \tilde{f}}\right) \phi_{p}=\sum_{p} \tilde{\gamma}_{p}^{R, \tilde{f}} \phi_{p}=\sum_{\nu} \tilde{\gamma}_{\nu}^{R, \tilde{f}} \chi_{\nu} \\
& \phi_{\tilde{f}}^{d, L}=\sum_{j, p} U_{\tilde{f} j}^{*}\left\langle\Psi_{j}^{L}\left|\hat{a}_{p}\right| \Psi_{0}^{R}\right\rangle \phi_{p}=\sum_{p}\left(\sum_{j} U_{\tilde{f} j}^{*} \gamma_{p}^{L, j}\right) \phi_{p}=\sum_{p} \tilde{\gamma}_{p}^{L, \tilde{f}} \phi_{p}=\sum_{\nu} \tilde{\gamma}_{\nu}^{L, \tilde{f}} \chi_{\nu} .
\end{aligned}
$$

where we introduced the amplitudes of the Dyson orbitals of the original non-relativistic EOM states ${ }^{24,61}$

$$
\gamma_{p}^{R, j}=\left\langle\Psi_{0}^{L}\left|\hat{a}_{p}^{\dagger}\right| \Psi_{j}^{R}\right\rangle ; \quad \gamma_{p}^{L, j}=\left\langle\Psi_{j}^{L}\left|\hat{a}_{p}\right| \Psi_{0}^{R}\right\rangle
$$

In the last equality of Eqs. (20) and (21), the Dyson orbitals are expressed on the atomic orbital basis $\left\{\chi_{\nu}\right\}$. The Dyson orbitals of the SO-split states are complex-valued. Complex orbitals can be visualized in different ways, from simply representing their real and imaginary components separately, to more sophisticated representations that take their phases into account. ${ }^{64,65}$ To visualize the complex Dyson orbitals, we use here the QSimulate-QM program, ${ }^{66}$ that implements the algorithm proposed in Ref. 65.

Fig. 2 shows zero-order and SO-coupled right Dyson orbitals for $\mathrm{H}_{2} \mathrm{~S}$, illustrating the effect of the SOC on the ionized states. SOC mixes the non-relativistic states and changes orbital shapes, i.e, $2 p_{1 / 2}$ and $2 p_{3 / 2}$ orbitals are rotated relative to the original $p_{x}, p_{y}$, and $p_{z}$, and also scrambles spin and space degrees of freedom. The SO-mixed orbitals transform by a different symmetry group (double point group), because the relativistic treatment necessitates using different symmetry groups, as described, for example, in Ref. 67,68. For a $\mathrm{C}_{2 v}$ molecule (such as $\mathrm{H}_{2} \mathrm{~S}$ ), the relativistic states belong to the $\bar{C}_{2 v}$ double group, which is a non-Abelian group with four one-dimensional irreps of the bosonic type and one two-dimensional irrep 
Figure 2: $\mathrm{H}_{2} \mathrm{~S}$. Left: right Dyson orbitals for non-relativistic EOM-IP states ( $\alpha$ and $\beta$ spin-orbitals have different signs, consistent with the phase treatment in Wigner-Eckart's theorem). Right: spin-integrated right Dyson orbitals of the SO-mixed states, represented as isosurfaces of their (complex) norm. ${ }^{65}$

of the fermionic type. Wave functions with odd and even number of electrons transform according to the fermionic and bosonic representations, respectively. The Dyson orbital spinors (Fig. 2) have the representation $E \times A_{1}=E$. This is a two-dimensional irrep, which corresponds to a Kramers doublet in the full symmetry group (with time-reversal operation). Thus, the apparent shape of the orbital depends on how the basis is selected in this irrep. In the SI, we present an alternative, symmetrized, rendering of the SO-mixed Dyson orbitals, together with the transformation matrix used to generate it, as well as the unconstrained transformation matrix $\mathbf{U}$.

These SO-mixed Dyson orbitals illustrate the effect of the SOC on the ionized states, in the same fashion as the analysis of the SO-mixed transition density matrices from Ref. 69 illustrates the effect of SOC on the excited states. We note that this analysis is based on the SO-mixed adiabatic states, in contrast to the SOC NTO analysis presented in Ref. 56, which is formulated in terms of the non-relativistic (diabatic) states.

The mixing also affects transition strengths, in the same fashion as it affects oscillator strengths. For example, the relative XPS intensities for the transitions involving the SO-split 
states are approximated as

$$
\tilde{\gamma}^{2}=\mathbf{U}^{\dagger} \gamma^{L} \gamma^{R} \mathbf{U}
$$

The SOC-CVS-EOM-CCSD approach has been implemented in the Q-Chem electronic structure package. ${ }^{70,71}$ The implementation included the extension of the fc-CVS-EOMCCSD framework to the SF states, in addition to the previously implemented ${ }^{23,24}$ IP and EE variants. For the XPS calculations, we used the $6-311+\mathrm{G}(3 d f)$ basis set with uncontracted core functions, denoted as $\mathrm{uC}-6-311+\mathrm{G}(3 d f)$, following the recommendation of a recent benchmark study. ${ }^{6}$ Because we focus on L-edges, we only uncontracted the core orbitals, roughly corresponding to $n=2$, i.e., the second contracted $s$-function (leaving the '6' contracted core function untouched) and the two most contracted $p$-functions. For the XAS calculations, we used uC-6-311 $(2+,+) \mathrm{G}(p, d)$ augmented with additional Rydberg-type functions whose exponents were generated according to the prescription of Kaufmann et al., ${ }^{72}$ and quantum number $n=2.5, \ldots, 5$. Uncontracted bases were used for the active edge only, whereas all other atoms were described by the standard variants of these basis sets. All basis sets are given in the SI. The number of states included in the SOMF Hamiltonian varies depending on the system, the exact number of states for each system can be found in SI.

To illustrate the capabilities of the method, we considered several systems for which experimental data are available. These systems are listed in Table ?? in the SI, along with their structural parameters. In most cases, we used structures optimized at the CCSD(T)/ccpCVQZ level of theory, taken from Ref. 73. For thiophene, we used an MP2/cc-pVTZ optimized structure. All Cartesian coordinates are given in the SI. 
Table 1: L-edge IEs $(\mathrm{eV})^{a}$ and SO splitting $\left(\mathrm{cm}^{-1}\right)$ for $\mathrm{H}_{2} \mathrm{~S}, \mathrm{OCS}, \mathrm{SO}_{2}, \mathrm{CS}_{2}$, and $\mathrm{C}_{4} \mathrm{H}_{4} \mathrm{~S}_{2}$.

a Theory: fc-CVS-EOMIP-CCSD/uC-6-311+G(3df). Experimental values are from Ref. 74 $\left(\mathrm{H}_{2} \mathrm{~S}, \mathrm{OCS}, \mathrm{SO}_{2}, \mathrm{CS}_{2}\right)$ and Ref. $75\left(\mathrm{C}_{4} \mathrm{H}_{4} \mathrm{~S}_{2}\right)$.

Table 1 shows the first three core-ionization energies (IEs) for $\mathrm{H}_{2} \mathrm{~S}, \mathrm{OCS}, \mathrm{SO}_{2}, \mathrm{CS}_{2}$, and $\mathrm{C}_{4} \mathrm{H}_{4} \mathrm{~S}_{2}$ computed with SOC-CVS-EOMIP-CCSD/uC-6-311+G(3df) and compares them with the experimental values. The table also shows the energy difference with respect to the first IE $(\Delta \mathrm{E})$. The corresponding zeroth-order energies, calculated at the non-relativistic fc-CVS-EOMIP-CCSD/uC-6-311+G(3df) level of theory, are given in Table S3 in the SI. The energies are assigned to the ionization of the electrons from the $2 p$ orbitals of the atom marked in bold. The results show how spin-orbit coupling splits the nearly degenerate $2 p$ orbitals into two sets: one $2 p_{1 / 2}$ orbital and two near-degenerate $2 p_{3 / 2}$ orbitals, as explained in Fig. 1 and illustrated in Fig. 2. The gap between these two sets is due to the SOC, whereas the small energy difference between the $2 p_{3 / 2}$ orbitals arises from non-spherically symmetric molecular environment (this splitting is called molecular field splitting). Depending on the 
system, the absolute deviations of the computed IEs relative to the experimental values are of the order of $0.1-1.0 \mathrm{eV}$, which corresponds to relative errors of the order of $0.05-0.5 \%$. Fig. 3 shows the computed XPS spectra of the thiophene molecule, illustrating the spectroscopic signatures of the SOC. At the non-relativistic level, the $2 p$ orbitals, although already slightly non-degenerate due to the molecular field, are still close enough so that the spectrum has only one peak. The inclusion of the SOC splits this peak into two, with the intensity ratio $2: 1$, corresponding to the ionization of two $2 p_{3 / 2}$ and the one $2 p_{1 / 2}$. After a shift of $+0.5 \mathrm{eV}$ the SO-corrected spectrum agrees well with the experiment, both in terms of the the intensity ratio and the energy splitting.

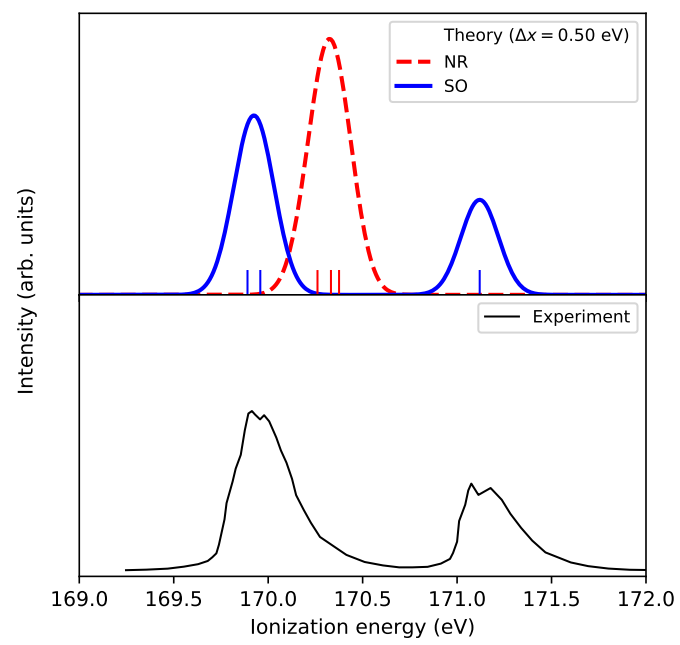

Figure 3: Thiophene $\left(\mathrm{C}_{4} \mathrm{H}_{4} \mathrm{~S}\right)$ L-edge XPS. The theoretical spectra were obtained using a Gaussian convolution function $(\sigma=0.15 \mathrm{eV})$ and an energy shift of $+0.5 \mathrm{eV}$, estimated by aligning to the first intense experimental peaks. The experimental spectrum was digitized from Ref. 76.

Fig. 4 compares the computed L-edge NEXAFS for $\mathrm{SiH}_{4}, \mathrm{SO}_{2}, \mathrm{C}_{4} \mathrm{H}_{4} \mathrm{~S}$, and $\mathrm{Ar}$ with the experimental spectra. The raw theoretical data (energies and oscillator strengths) are provided in the SI.

Fig. 4a shows the L-edge spectra of silane with and without SOCs. As in the XPS example above, the first peak is split into two upon inclusion of the SOC and agrees well with the 


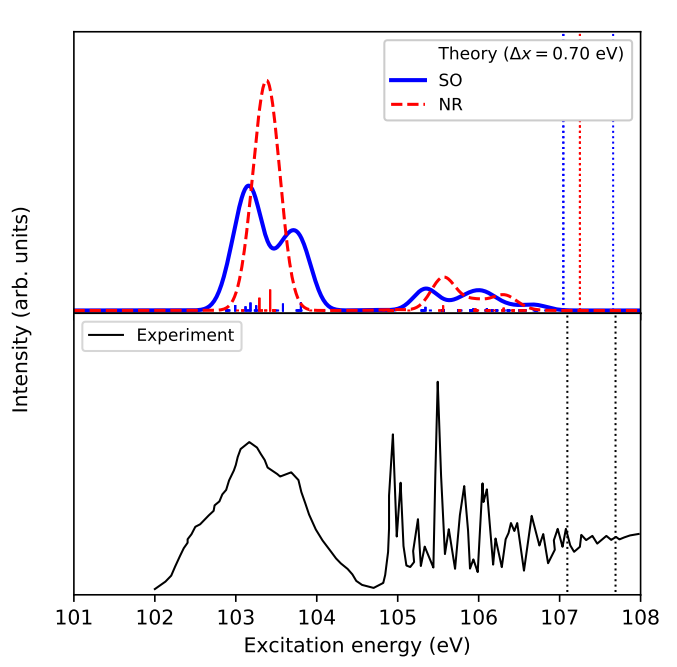

(a) $\mathrm{SiH}_{4}$

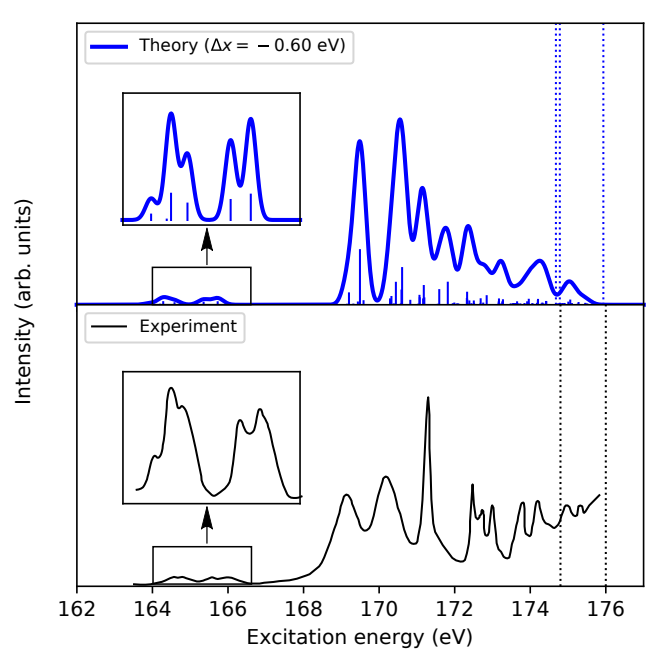

(c) $\mathrm{SO}_{2}$

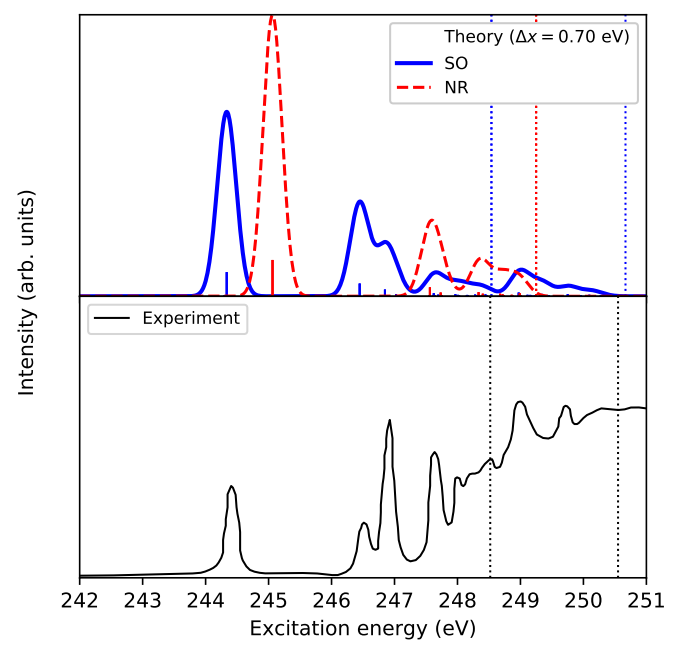

(b) $\mathrm{Ar}$

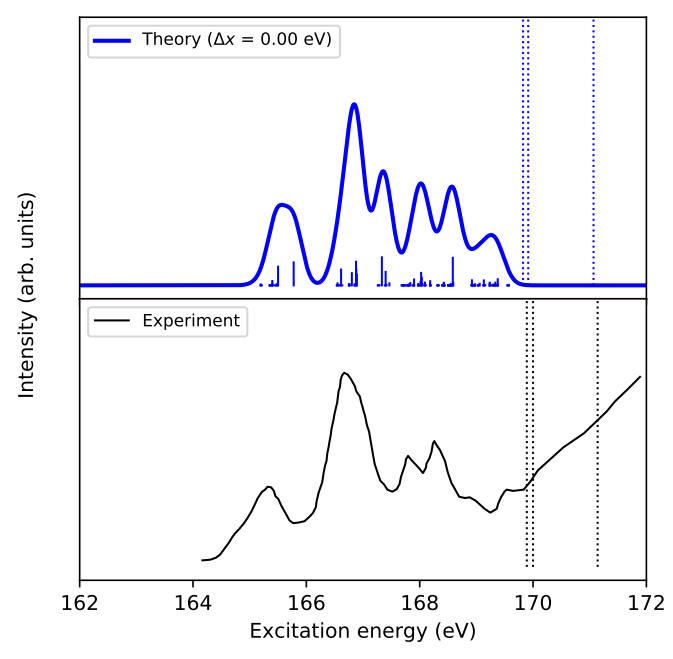

(d) Thiophene, $\mathrm{C}_{4} \mathrm{H}_{4} \mathrm{~S}$

Figure 4: L-edge XAS of $\mathrm{SiH}_{4}(4 \mathrm{a}), \mathrm{Ar}(4 \mathrm{~b}), \mathrm{SO}_{2}(4 \mathrm{c})$, and $\mathrm{C}_{4} \mathrm{H}_{4} \mathrm{~S}(4 \mathrm{~d})$. The theoretical spectra were obtained using a Gaussian convolution function $(\sigma=0.15 \mathrm{eV})$ and an energy shift $(\Delta x)$ for a better fit with experiment. The experimental spectra were digitized from Ref. 77 for $\mathrm{SiH}_{4}$, Ref. 78 for Ar, Ref. 79 for $\mathrm{SO}_{2}$ and Ref. 75 for thiophene. The vertical dotted lines correspond to the $\mathrm{L}_{2}$ and $\mathrm{L}_{3}$ ionization energies. The label NR denotes non-relativistic calculations without inclusion of the SOC.

experiment. The shifted IEs are also in good agreement with the experiment. However, our convoluted spectrum does not reproduce the highly structured set of bands above $104.4 \mathrm{eV}$ observed in the experiment, possibly due to using the same empirical Gaussian broadening 
function for all computed states, regardless of their actual lifetime.

Fig. $4 \mathrm{~b}$ shows the spectra for the argon atom. Theory and experiment agree well, after a small shift of only $+0.7 \mathrm{eV}$ is applied. The first band at around $244.5 \mathrm{eV}$ is due to the $2 p_{3 / 2} \rightarrow 4 s$ transition, whereas the second band at around $246.5 \mathrm{eV}$ corresponds to the $2 p_{1 / 2} \rightarrow 4 s$ transition. The third and forth bands contain contributions from the $2 p_{3 / 2} \rightarrow 5 s, 3 d$ and $2 p_{1 / 2} \rightarrow 5 s, 3 d$ transitions, respectively. In the case of sulfur dioxide (see Fig. 4c) the agreement between theory and experiment is also quite good for the first peaks (zoomed-in region), after a shift of $-0.6 \mathrm{eV}$ is applied. The agreement deteriorates slightly at higher energies.

Finally, Fig. 4d illustrates the spectra for a larger molecule, thiophene. In this case, the theoretical spectrum is in excellent agreement with the experimental one in the entire energy range and without an energy shift.

In conclusion, we have presented the first implementation of the perturbative inclusion of spin-orbit effects within coupled-cluster theory to describe core-level spectroscopy. This has been achieved by utilizing a general framework for calculating the SOCs from spinless one-particle density matrices computed for the fc-CVS-EOM-CCSD wave functions. This methodological advance enables the calculation of SO-corrected ionization and excitation energies by a simple two-step procedure. In the first step, the non-relativistic states are computed using appropriate variants of the EOM-CC family of methods; the choice of the method is determined by the target states, i.e., EOM-IP for ionized states and EOM-EE/SF for excited states. In the second step, these zeroth-order states are mixed by the perturbation due to the SO part of the Breit-Pauli Hamiltonian, giving rise to the SO-corrected energies and intensities. The examples illustrate the capabilities of the new method to accurately and efficiently simulate L-edge XAS and XPS. 


\section{Acknowledgement}

The authors thank Dr. Toru Shiozaki from Quantum Simulation Technologies, Inc. for his help with the visualization of complex orbitals and for helpful discussions. P. P. thanks Dr. Dmitri Bezrukov from the Lomonosov Moscow State University for his insightful comments on relativistic symmetry. S.C. thanks Professor Lan Cheng from the Johns Hopkins University for valuable discussions during the initial phase of this project. M.L.V. and S.C. acknowledge financial support from DTU Chemistry (Ph.D. grant to M.L.V) and from the Independent Research Fund Denmark-Natural Sciences (Research-Project-2 grant No. 7014-00258B to S.C.). The work at USC was supported by the U.S. National Science Foundation (No. CHE-1856342 to A.I.K.).

\section{Note}

The authors declare the following competing financial interest(s): A. I. K. is the president and a part-owner of Q-Chem, Inc.

\section{Supporting Information Available}

Additional information: Symmetrized Dyson orbitals for $\mathrm{H}_{2} \mathrm{~S}$; Cartesian coordinates; Basis sets; zeroth-order IEs and spectroscopic data (raw data for XAS of Ar, $\mathrm{H}_{2} \mathrm{~S}, \mathrm{OCS}, \mathrm{SO}_{2} \mathrm{SiH}_{4}$ and $\mathrm{C}_{4} \mathrm{H}_{4} \mathrm{~S}$, and XAS spectra of OCS and $\mathrm{H}_{2} \mathrm{~S}$ ).

\section{References}

(1) van Bokhoven, J. A., Lamberti, C., Eds. X-Ray Absorption and X-ray Emission Spectroscopy; Theory and Applications; Wiley \& Sons, 2016. 
(2) Mobilio, S., Boscherini, F., Meneghini, C., Eds. Synchrotron Radiation: Basics, Methods and Applications; Springer, 2014.

(3) Bergmann, U., Yachandra, V. K., Yano, J., Eds. X-Ray Free Electron Lasers: Applications in Materials, Chemistry and Biology; Energy and Environment Series 18; Royal Society of Chemistry, 2017.

(4) Norman, P.; Dreuw, A. Simulating X-ray Spectroscopies and Calculating Core-Excited States of Molecules. Chem. Rev. 2018, 118, 7208-7248.

(5) Carbone, J. P.; Cheng, L.; Myhre, R. H.; Matthews, D.; Koch, H.; Coriani, S. Chapter Eleven - An analysis of the performance of coupled cluster methods for K-edge core excitations and ionizations using standard basis sets. In State of The Art of Molecular Electronic Structure Computations: Correlation Methods, Basis Sets and More; Ancarani, L. U., Hoggan, P. E., Eds.; Adv. Quantum Chem.; Academic Press, 2019; Vol. 79; pp $241-261$.

(6) Sarangi, R.; Vidal, M. L.; Coriani, S.; Krylov, A. I. On the basis set selection for calculations of core-level states: different strategies to balance cost and accuracy. Mol. Phys. 2020, 0, e1769872.

(7) Sekino, H.; Bartlett, R. J. A linear response, coupled-cluster theory for excitation energy. Int. J. Quant. Chem. 1984, 26, 255-265.

(8) Stanton, J. F.; Bartlett, R. J. The equation of motion coupled-cluster method. A systematic biorthogonal approach to molecular excitation energies, transition probabilities, and excited state properties. J. Chem. Phys. 1993, 98, 7029-7039.

(9) Krylov, A. I. Equation-of-Motion Coupled-Cluster Methods for Open-Shell and Electronically Excited Species: The Hitchhiker's Guide to Fock Space. Ann. Rev. Phys. Chem. 2008, 59, 433-462. 
(10) Bartlett, R. J. Coupled-cluster theory and its equation-of-motion extensions. WIREs Comput Mol Sci 2012, 2, 126-138.

(11) Sneskov, K.; Christiansen, O. Excited state coupled cluster methods. WIREs Comput. Mol. Sci. 2012, 2, 566-584.

(12) Coriani, S.; Pawłowski, F.; Olsen, J.; Jørgensen, P. Molecular response properties in equation of motion coupled cluster theory: A time-dependent perspective. J. Chem. Phys. 2016, 144, 024102.

(13) Sadybekov, A.; Krylov, A. I. Coupled-cluster based approach for core-ionized and coreexcited states in condensed phase: Theory and application to different protonated forms of aqueous glycine. J. Chem. Phys. 2017, 147, 014107.

(14) Faber, R.; Coriani, S. Corevalence-separated coupled-cluster-singles-and-doubles complex-polarization-propagator approach to X-ray spectroscopies. Phys. Chem. Chem. Phys. 2020, 22, 2642.

(15) Cederbaum, L. S.; Domcke, W.; Schirmer, J. Many-body theory of core holes. Phys. Rev. A: At. Mol. Opt. Phys. 1980, 22, 206-222.

(16) Barth, A.; Schirmer, J. Theoretical core-level excitation spectra of $\mathrm{N}_{2}$ and CO by a new polarisation propagator method. J. Phys. B: At. Mol. Phys. 1985, 18, 867-885.

(17) Trofimov, A. B.; Moskovskaya, T. E.; Gromov, N. M., E. V. Vitkovskaya; Schirmer, J. Core-level electronic spectra in $\mathrm{ADC}(2)$ approximation for polarization propagator: carbon monoxide and nitrogen molecules. J. Struct. Chem. 2000, 41, 483-494.

(18) Stener, M.; Fronzoni, G.; de Simone, M. Time Dependent Density Functional Theory of Core Electrons Excitations. Chem. Phys. Lett. 2003, 15, 115.

(19) Wenzel, J.; Wormit, M.; Dreuw, A. Calculating Core-Level Excitations and X-Ray Absorption Spectra of Medium-Sized Closed-Shell Molecules with the Algebraic- 
Diagrammatic Construction Scheme for the Polarization Propagator. J. Comput. Chem. 2014, 35, 1900 .

(20) Wenzel, J.; Holzer, A.; Wormit, M.; Dreuw, A. Analysis and Comparison of CVS-ADC Approaches up to Third Order for the Calculation of Core-Excited States. J. Chem. Phys. 2015, 142, 214104.

(21) Delcey, M. G.; Srensen, L. K.; Vacher, M.; Couto, R. C.; Lundberg, M. Efficient calculations of a large number of highly excited states for multiconfigurational wavefunctions. J. Comput. Chem. 2019, 40, 1789-1799.

(22) Coriani, S.; Koch, H. Communication: X-ray Absorption Spectra and Core-Ionization Potentials within a Core-Valence Separated Coupled Cluster Framework. J. Chem. Phys. 2015, 143, 181103.

(23) Vidal, M. L.; Feng, X.; Epifanovsky, E.; Krylov, A. I.; Coriani, S. New and Efficient Equation-of-Motion Coupled-Cluster Framework for Core-Excited and Core-Ionized States. J. Chem. Theory Comput. 2019, 15, 3117-3133.

(24) Vidal, M. L.; Krylov, A. I.; Coriani, S. Dyson orbitals within the fc-CVS-EOM-CCSD framework: theory and application to X-ray photoelectron spectroscopy of ground and excited states. Phys. Chem. Chem. Phys. 2020, 22, 2693.

(25) Frati, F.; De Groot, F.; Cerezo, J.; Santoro, F.; Cheng, L.; Faber, R.; Coriani, S. Coupled cluster study of the x-ray absorption spectra of formaldehyde derivatives at the oxygen, carbon, and fluorine K-edges. J. Chem. Phys. 2019, 151, 064107.

(26) Myhre, R. H.; Coriani, S.; Koch, H. Near-edge X-ray absorption fine structure within multilevel coupled cluster theory. J. Chem. Theory Comput. 2016, 12, 2633-2643.

(27) Liu, J.; Matthews, D.; Coriani, S.; Cheng, L. Benchmark Calculations of K-Edge Ionization Energies for First-Row Elements Using Scalar-Relativistic Core-Valence-Separated 
Equation-of-Motion Coupled-Cluster Methods. J. Chem. Theory Comput. 2019, 15, $1642-1651$.

(28) Tenorio, B. N. C.; Moitra, T.; Nascimento, M. A. C.; Rocha, A. B.; Coriani, S. Molecular inner-shell photoabsorption/photoionization cross sections at core-valence-separated coupled cluster level: Theory and examples. J. Chem. Phys. 2019, 150, 224104.

(29) Faber, R.; Coriani, S. Resonant Inelastic X-ray Scattering and Nonesonant X-ray Emission Spectra from Coupled-Cluster (Damped) Response Theory. J. Chem. Theory Comput. 2019, 15, 520-528.

(30) Nanda, K.; Vidal, M. L.; Faber, R.; Coriani, S.; Krylov, A. I. How to stay out of trouble in RIXS calculations within the equation-of-motion coupled-cluster damped response theory framework? Safe hitchhiking in the excitation manifold by means of core-valence separation. Phys. Chem. Chem. Phys. 2020, 22, 2629.

(31) Nanda, K.; Krylov, A. I. A simple molecular orbital picture of RIXS distilled from many-body damped response theory. J. Chem. Phys. 2020, in press.

(32) Pyykko, P. Relativistic effects in structural chemistry. Chem. Rev. 1988, 88, 563-594.

(33) Coriani, S.; Christiansen, O.; Fransson, T.; Norman, P. Coupled-Cluster Response Theory for Near-Edge X-Ray-Absorption Fine Structure of Atoms and Molecules. Phys. Rev. A 2012, 85, 022507.

(34) Saue, T. Relativistic Hamiltonians for chemistry: A primer. ChemPhysChem 2011, 12, $3077-3094$.

(35) Visscher, L.; Lee, T. J.; Dyall, K. G. Formulation and implementation of a relativistic unrestricted coupled-cluster method including noniterative connected triples. J. Chem. Phys. 1996, 105, 8769-8776. 
(36) Shee, A.; Visscher, L.; Saue, T. Analytic one-electron properties at the 4-component relativistic coupled cluster level with inclusion of spin-orbit coupling. J. Chem. Phys. 2016, $145,184107$.

(37) Shee, A.; Saue, T.; Visscher, L.; Gomes, A. S. P. Equation-of-motion coupled-cluster theory based on the 4-component Dirac-Coulomb(-Gaunt) Hamiltonian. Energies for single electron detachment, attachment, and electronically excited states. J. Chem. Phys. 2018, 149, 174113.

(38) Chang, C.; Pelissier, M.; Durand, P. Regular two-component Pauli-like effective Hamiltonians in Dirac theory. Physica Scripta 1986, 34, 394.

(39) Lenthe, E. v.; Baerends, E.-J.; Snijders, J. G. Relativistic regular two-component Hamiltonians. J. Chem. Phys. 1993, 99, 4597-4610.

(40) van Lenthe, E.; Baerends, E.-J.; Snijders, J. G. Relativistic total energy using regular approximations. J. Chem. Phys. 1994, 101, 9783-9792.

(41) Van Lenthe, E.; Snijders, J. G.; Baerends, E. J. The zero-order regular approximation for relativistic effects: The effect of spin-orbit coupling in closed shell molecules. J. Chem. Phys. 1996, 105, 6505-6516.

(42) Dyall, K. G.; van Lenthe, E. Relativistic regular approximations revisited: An infiniteorder relativistic approximation. J. Chem. Phys. 1999, 111, 1366-1372.

(43) Douglas, M.; Kroll, N. M. Quantum electrodynamical corrections to the fine structure of helium. Annals of Physics 1974, 82, 89-155.

(44) Hess, B. A. Relativistic electronic-structure calculations employing a two-component no-pair formalism with external-field projection operators. Phys. Rev. A 1986, 33, 3742.

(45) Jansen, G.; Heß, B. A. Revision of the Douglas-Kroll transformation. Phys. Rev. A 1989, 39, 6016. 
(46) Iliaš, M.; Saue, T. An infinite-order two-component relativistic Hamiltonian by a simple one-step transformation. J. Chem. Phys. 2007, 126, 064102.

(47) Filatov, M.; Zou, W.; Cremer, D. Spin-orbit coupling calculations with the twocomponent normalized elimination of the small component method. J. Chem. Phys. 2013, 139, 014106.

(48) Cheng, L.; Wang, F.; Stanton, J. F.; Gauss, J. Perturbative treatment of spinorbit-coupling within spin-free exact two-component theory using equation-of-motion coupled-cluster methods. J. Chem. Phys. 2018, 148, 044108.

(49) Fleig, T. Time-reversal symmetry in general coupled cluster theory. Phys. Rev. A 2008, 7r, 062503.

(50) Wahlgren, U.; Sjøvoll, M.; Fagerli, H.; Gropen, O.; Schimmelpfennig, B. Ab initio calculations of the ${ }^{2} P_{1 / 2}-{ }^{2} P_{3 / 2}$ splitting in the thallium atom. Theor. Chem. Acc. 1997, 97, 324-330.

(51) Christiansen, O.; Gauss, J.; Schimmelpfennig, B. Spin-orbit coupling constants from coupled-cluster response theory. Phys. Chem. Chem. Phys. 2000, 2, 965-971.

(52) Klein, K.; Gauss, J. Perturbative calculation of spin-orbit splittings using the equationof-motion ionization-potential coupled-cluster ansatz. J. Chem. Phys. 2008, 129, 194106.

(53) Mück, L. A.; Gauss, J. Communication: Spin-orbit splittings in degenerate open-shell states via Mukherjee's multireference coupled-cluster theory: A measure for the coupling contribution. J. Chem. Phys. 2012, 136, 111103.

(54) Epifanovsky, E.; Klein, K.; Stopkowicz, S.; Gauss, J.; Krylov, A. I. Spin-orbit couplings within the equation-of-motion coupled-cluster framework: Theory, implementation, and benchmark calculations. J. Chem. Phys. 2015, 143, 064102. 
(55) Pokhilko, P.; Epifanovsky, E.; Krylov, A. I. General framework for calculating spinorbit couplings using spinless one-particle density matrices: Theory and application to the equation-of-motion coupled-cluster wave functions. J. Chem. Phys. 2019, 151, 034106.

(56) Pokhilko, P.; Krylov, A. I. Quantitative El-Sayed Rules for Many-Body Wavefunctions from Spinless Transition Density Matrices. J. Phys. Chem. Lett. 2019, 10, 4857-4862.

(57) Reiher, M.; Wolf, A. Relativistic quantum chemistry: the fundamental theory of molecular science; John Wiley \& Sons, 2014.

(58) Helgaker, T.; Coriani, S.; Jørgensen, P.; Kristensen, K.; Olsen, J.; Ruud, K. Recent Advances in Wave Function-Based Methods of Molecular-Property Calculations. Chem. Rev. 2012, 112, 543-631.

(59) Hess, B. A.; Marian, C. M.; Wahlgren, U.; Gropen, O. A mean-field spin-orbit method applicable to correlated wavefunctions. Chem. Phys. Lett. 1996, 251, 365-371.

(60) Schimmelpfennig, B. AMFI, An atomic mean-field spin-orbit integral program. 1996.

(61) Oana, C. M.; Krylov, A. I. Dyson orbitals for ionization from the ground and electronically excited states within equation-of-motion coupled-cluster formalism: Theory, implementation, and examples. J. Chem. Phys. 2007, 127, 234106.

(62) Oana, C. M.; Krylov, A. I. Cross sections and photoelectron angular distributions in photodetachment from negative ions using equation-of-motion coupled-cluster Dyson orbitals. J. Chem. Phys. 2009, 131, 124114.

(63) Moitra, T.; Ponzi, A.; Koch, H.; Coriani, S.; Decleva, P. Accurate Description of Photoionization Dynamical Parameters. J. Phys. Chem. Lett. 2020, 11, 5330-5337.

(64) Asher, J. R. An animated visualization of orbital angular momentum and spin-orbit coupling. Int. J. Quantum Chem. 2018, 118, e25683. 
(65) Al-Saadon, R.; Shiozaki, T.; Knizia, G. Visualizing Complex-Valued Molecular Orbitals. J. Phys. Chem. A 2019, 123, 3223-3228.

(66) QSimulate-QM, Quantum Simulation Technologies, Inc. 2020.

(67) Zaitsevskii, A. Relyativiskaya teoriya electronnogo stroyeniya molekul; M.V. Lomonosov Moscow State University, Moscow, 2005.

(68) Peng, D.; Ma, J.; Liu, W. On the construction of Kramers paired double group symmetry functions. Int. J. Quant. Chem. 2009, 109, 2149-2167.

(69) Mai, S.; Plasser, F.; Dorn, J.; Fumanal, M.; Daniel, C.; González, L. Quantitative wave function analysis for excited states of transition metal complexes. Coord. Chem. Rev. 2018, 361, 74-97.

(70) Krylov, A. I.; Gill, P. M. W. Q-Chem: An engine for innovation. WIREs Comput. Mol. Sci. 2013, 3, 317-326.

(71) Shao, Y.; Gan, Z.; Epifanovsky, E.; Gilbert, A. T.; Wormit, M.; Kussmann, J.; Lange, A. W.; Behn, A.; Deng, J.; Feng, X. et al. Advances in molecular quantum chemistry contained in the Q-Chem 4 program package. Mol. Phys. 2015, 113, 184215.

(72) Kaufmann, K.; Baumeister, W.; Jungen, M. Universal Gaussian basis sets for an optimum representation of Rydberg and continuum wavefunctions. J. Phys. B: At., Mol. Opt. Phys. 1989, 22, 2223.

(73) Coriani, S.; Marchesan, D.; Gauss, J.; Hättig, C.; Helgaker, T.; Jørgensen, P. The accuracy of ab initio molecular geometries for systems containing second-row atoms. J. Chem. Phys. 2005, 123, 184107.

(74) Coville, M.; Thomas, T. Sulfur 2p ionization energies of H2S, OCS, SO2, and CS2. Journal of Electron Spectroscopy and Related Phenomena 1995, 71, 21-23. 
(75) Baseggio, O.; Toffoli, D.; Stener, M.; Fronzoni, G.; de Simone, M.; Grazioli, C.; Coreno, M.; Guarnaccio, A.; Santagata, A.; DAuria, M. S2p core level spectroscopy of short chain oligothiophenes. J. Chem. Phys. 2017, 147, 244301.

(76) Siggel, M. R. F.; Field, C.; Sæthre, L. J.; Børve, K. J.; Thomas, T. D. High resolution photoelectron spectroscopy of sulfur 2 p electrons in $\mathrm{H}_{2} \mathrm{~S}, \mathrm{SO}_{2}, \mathrm{CS}_{2}$, and OCS. J. Chem. Phys. 1996, 105, 9035-9039.

(77) Püttner, R.; Domke, M.; Lentz, D.; Kaindl, G. Si $2 p$ photoabsorption in $\mathrm{SiH}_{4}$ and $\mathrm{SiD}_{4}$ : Molecular distortion in core-excited silane. Phys. Rev. A 1997, 56, 1228-1239.

(78) Nakamura, M.; Sasanuma, M.; Sato, S.; Watanabe, M.; Yamashita, H.; Iguchi, Y.; Ejiri, A.; Nakai, S.; Yamaguchi, S.; Sagawa, T. et al. Absorption Structure Near the $\mathrm{L}_{I I, I I I}$ Edge of Argon Gas. Phys. Rev. Lett. 1968, 21, 1303.

(79) Krasnoperova, A. A.; Gluskin, E. S.; Mazalov, L. N.; Kochubei, V. A. The fine structure of the $\mathrm{L}_{I I, I I I}$ absorption edge of sulfur in the $\mathrm{SO}_{2}$ molecule. J. Struct. Chem. 1976, 17, $947-950$. 